\title{
Broadband tuning of polarization modulation instability in microstructured optical fibers
}

\author{
A. Loredo-Trejo ${ }^{1,2}$, A. Díez ${ }^{1,2}$, E. Silvestre ${ }^{1,3}$, M. V. Andrés $S^{1,2}$ \\ ${ }^{1}$ Laboratory of Fiber Optics, ICMUV, Universidad de Valencia, Dr. Moliner 50, 46100, Burjassot, Spain \\ ${ }^{2}$ Departamento de Física Aplicada y Electromagnetismo, Universidad de Valencia, Dr. Moliner 50, 46100, Burjassot, Spain \\ ${ }^{3}$ Departamento de Óptica, Universidad de Valencia, Dr. Moliner 50, 46100, Burjassot, Spain \\ *Corresponding author: antonio.diez@uv.es
}

Received XX Month XXXX; revised XX Month, XXXX; accepted XX Month XXXX; posted XX Month XXXX (Doc. ID XXXXX); published XX Month XXXX

\begin{abstract}
Wideband tuning of strong bands generated through polarization modulation instability (PMI) in microstructured optical fibers (MOFs) is reported. Tunability is achieved by exploiting the dependence of the phase-matching condition on the fiber's chromatic dispersion and birefringence, which is particularly sensitive when the fiber is pumped near the zerodispersion wavelength. MOFs designed to accomplish PMI phase-matching when they are infiltrated with ethanol and pumped at $1064 \mathrm{~nm}$ were designed and fabricated. Taking advantage of the large thermo-optic coefficient of ethanol, both chromatic dispersion and birefringence were varied through temperature. Wavelength shifts from $937 \mathrm{~nm}$ to $863 \mathrm{~nm}$ (anti-Stokes) and from $1231 \mathrm{~nm}$ to $1387 \mathrm{~nm}$ (Stokes) is demonstrated. Such a tuning range corresponds to a frequency shift between the pump and a PMI sideband ranging from $1274 \mathrm{~cm}^{-1}$ to $2189 \mathrm{~cm}^{-1}$.
\end{abstract}

\section{(C) 2019 Optical Society of America}

http://dx.doi.org/10.1364/OL.99.099999

Four-wave mixing/modulation instability (FWM/MI) is a versatile optical nonlinear parametric process in which two pump photons are transferred to symmetric sidebands whose spectral location is set by the requirements of energy and momentum conservation. Despite the low nonlinearity of silica, optical fibers are a good platform for the generation of nonlinear effects, including FWM/MI, due to the strong optical field density, the viability of long interaction lengths, and the control of chromatic dispersion. Additionally, the development of microstructured optical fibers (MOF) benefiting from higher nonlinearity and greater flexibility for chromatic dispersion design than conventional fibers, has broaden the potentiality of optical fibers for nonlinear applications. Over the last decades, a large number of all-optical fiber devices based on FWM/MI have been demonstrated, such as parametric oscillators and amplifiers [1], and new light sources for specific applications such as correlated photon-pair generation [2] and coherent Raman scattering microscopy [3-4].

Depending on the polarization state of the involved photons, a distinction is usually made between scalar FWM/MI processes, in which the polarization of the generated photons is identical to that of the pump photons, and vector processes, which involves the interaction between photons with different polarization states [5]. Among the latter, we find two different forms, polarization modulation instability (PMI) and cross-phase modulation instability (XPMI). XPMI can be observed in optical fibers with moderate-to-high values of birefringence, so that a linearly polarized pump oriented at $45^{\circ}$ with respect to the main axes of the fiber generates two orthogonally polarized sidebands, each one being polarized along one the main axes of the fiber. The second form, PMI, can be produced in very weakly birefringent fibers (or even in isotropic fibers). The polarization of the two generated bands is the same, and orthogonal to the polarization of the pump. PMI features are quite different depending on whether the pump is polarized along the slow or the fast axis of the fiber. In the first case (slow-axis pump), PMI produces sidebands separated from the pump regardless of the pump power. For fast-axis pumping, a low-power threshold exists, the gain profile only shows sidebands detuned from the pump at high pump powers, and the gain at zero detuning does not vanish regardless of the pump power level. The first experimental observation of PMI in lowbirefringence optical fibers was reported in 1995 [6]. Experiments were performed with a conventional single-mode fiber whose chromatic dispersion was normal at the pump wavelength. In the context of MOFs, PMI was first observed in the normal dispersion regime in a large-air-filling fraction MOF pumped near the zerodispersion wavelength (ZDW) [7]. Recently, we demonstrated PMI generation in all-normal dispersion MOFs [8].

In many applications based on FWM/MI, the ability of tuning the generated light is a prime requirement. Different strategies have been followed in the past to tune the bands produced by scalar FWM/MI, as for instance, by tuning the pump laser wavelength [9]. Alternatively, tunability can be achieved by varying the chromatic 
dispersion of the fiber. In previous works, we demonstrated large tuning ranges using liquid-infiltrated MOFs designed to produce widely-spaced scalar FWM bands when pumped at $1064 \mathrm{~nm}$ [1011]. The chromatic dispersion of such infiltrated fibers, and therefore the spectral location of the generated bands, were tuned by changing the refractive index of the filling liquids by taking advantage of their large thermo-optic coefficient.

The effect of PMI in optical fibers can be analyzed by a vector theory of four-wave mixing [12], where the polarization state of light and the fiber birefringence are taken into account. The net phase mismatch and the energy conservation relations can be used to calculate the frequency shift of the sidebands. The phasematching condition, for the two PMI processes are given by,

$$
\begin{aligned}
& 2 \beta_{p}-\beta_{A S}-\beta_{S}-\frac{\Delta n \cdot\left(\omega_{A S}+\omega_{S}\right)}{c}+\frac{2}{3} \gamma P=0 \\
& 2 \beta_{p}-\beta_{A S}-\beta_{S}+\frac{\Delta n \cdot\left(\omega_{A S}+\omega_{S}\right)}{c}+\frac{2}{3} \gamma P=0
\end{aligned}
$$

where $\beta_{p}, \beta_{A S}, \beta_{S}$ are the propagation factor of the fiber mode at the pump, anti-Stokes, and Stokes wavelengths, respectively, $\Delta n$ is the phase birefringence, $\omega_{A S}, \omega s$ are the frequencies of the generated photons, $c$ is the speed of light in vacuum, $P$ is the pump power, and $\gamma$ is the nonlinear coefficient of the fiber that is given by $\gamma=n_{2} \cdot \omega_{p} / c \cdot A_{\text {eff }}$ where $n_{2}$ is the nonlinear refractive index of silica, $\omega_{p}$ is the frequency of the pump, and $A_{\text {eff }}$ is the effective mode area. Eq. (1) corresponds to the case when the polarization of the pump photons is aligned to the fast axis of the fiber and the generated photons are polarized parallel to the slow axis, and Eq. (2), the opposite. Eqs. (1)-(2) together with conservation of energy determine the frequencies of the parametric bands in both cases. It can be seen that both, the fiber chromatic dispersion and the birefringence contribute to the linear phase, thus fulfillment of the phase-matching condition depends on both parameters. In previous reports, the frequency shift of the sidebands generated by PMI was tuned successfully through the variation of the fiber birefringence. Tuning ranges of few $\mathrm{THz}$ were demonstrated in conventional optical fibers pumped in the slow axis, in which birefringence was varied through different external factors such as bending or temperature $[5,6]$. Alternatively, tunability of PMI can be achieved by changing the fiber dispersion, similarly as it was done in $[10,11]$ for the scalar FWM. It happens that the dependence of PMI frequency detuning on the fiber dispersion and on birefringence is particularly notable for low values of dispersion $[5,6]$. Therefore, PMI wavelengths can be very sensitive to small changes of these two fiber parameters when the pump wavelength is close to ZDW of the fiber [7].

In this letter, we demonstrate experimentally, for the first time, broadband tuning of widely spaced PMI bands generated by pumping in normal dispersion near the ZDW, by simultaneous variation of fiber dispersion and birefringence.

We performed the experiments with ethanol filled solid-core MOFs. The fiber properties were varied by changing the refractive index of ethanol through temperature, as in $[10,11]$. MOFs were designed and fabricated with the appropriate characteristics to produce widely spaced PMI bands for slow-axis pumping when the holes were filled with ethanol. Fig. 1(a) shows the dispersion properties at room temperature $\left(20^{\circ} \mathrm{C}\right)$ of one of the fibers investigated, both with the holes empty and filled with ethanol. The fiber has a pitch $\Lambda=3.97 \mu \mathrm{m}$ and a normalized diameter of holes $d / \Lambda=0.75$. Dots are the experimental dispersion values and lines are the theoretical calculation. Theoretical modelling of the MOF was performed by taking into account the actual spatial refractive-index distribution obtained from SEM images of the fibers cross sections [13]. Modal indices, phase birefringence and effective area were calculated. The wavelength dispersion of refractive indices of silica and ethanol [14] were taken into account in the simulations. The fiber exhibits a small residual phase birefringence $\Delta n$ due to slight imperfections of the fiber microstructure, $\Delta n=2.0 \times 10^{-5}$ for the ethanol infiltrated fiber at room temperature.

Fig. 1(b) shows the calculated phase matching wavelengths for scalar FWM/MI and for slow-axis pump PMI, as a function of pump wavelength. A value of $\mathrm{n}_{2}=3.2 \times 10^{-20} \mathrm{~m}^{2} / \mathrm{W}$ was assumed in the calculations. When the holes are with air, no solutions were found for PMI at the pump wavelength of $1064 \mathrm{~nm}$. Only scalar MI can be generated. This was observed experimentally, and it is shown in the inset of Fig. 4. When the holes are with ethanol, the ZDW shifts to $1119 \mathrm{~nm}$, and the chromatic dispersion at $1064 \mathrm{~nm}$ switches from anomalous to normal. There are no solutions for scalar FWM/MI now at $1064 \mathrm{~nm}$, instead two solutions for PMI are found.
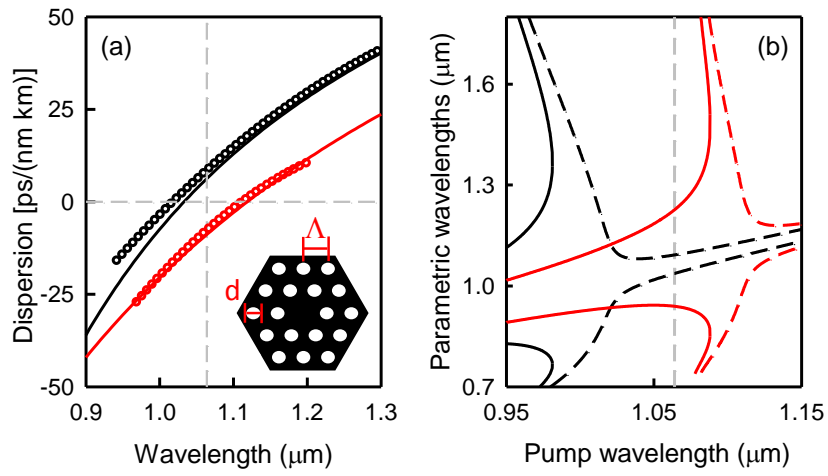

Fig. 1. (a) MOF chromatic dispersion as a function of wavelength, filled with air (black) and with ethanol (red). Dots are the experimental measurements and lines are the theoretical calculations. (b) Calculated slow-axis pumping PMI (solid lines) and scalar FWM/MI (dashed line) phase-matching curves of the pristine fiber (black) and the ethanolinfiltrated fiber $(\mathrm{P}=1 \mathrm{~kW})$. Vertical grey dashed line indicates the experimental pump wavelength.
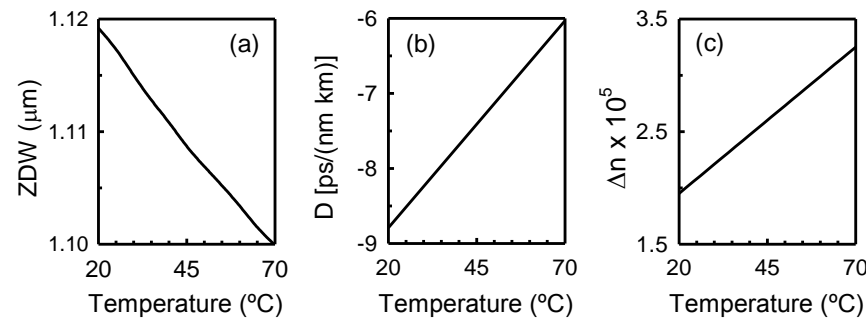

Fig. 2. (a) Zero-dispersion wavelength, (b) chromatic dispersion at $1064 \mathrm{~nm}$, and (c) phase birefringence at $1064 \mathrm{~nm}$, as a function of temperature, for the fiber infiltrated with ethanol.

The guiding properties of the fiber infiltrated with ethanol change with temperature. Owing to the large thermo-optic 
coefficient of ethanol $\left(-3.94 \times 10^{-4} / \mathrm{K}\right.$ [15]), small changes in temperature lead to significant changes in modal dispersion, phase birefringence, and effective area. In particular, the ZDW shifts to shorter wavelengths and, consequently, the modal dispersion at $1064 \mathrm{~nm}$ decreases, as shown in Fig. 2(a)-(b). The phase birefringence increases with temperature as a result of the increment of refractive index contrast between silica and holes owing to the negative thermo-optic coefficient of ethanol (see Fig. 2(c)). Figure 3 shows the PMI phase-matching wavelengths for different temperatures. As the temperature increases, the PMI wavelengths shift away from the pump wavelength. Notice that PMI frequency shift increases with temperature, thus, the temperature response is opposite to what it was shown for scalar FWM $[10,11]$. Due to the curve bending, there is a threshold temperature above which no solutions are found for a given pump wavelength $\left(\sim 65^{\circ} \mathrm{C}\right.$ at $1064 \mathrm{~nm}$ for this specific fiber).

The decrease of chromatic dispersion at the pump wavelength with temperature, and the increase of phase birefringence give independently rise to an increment of the PMI frequency shift. Thus both add up and contribute together to enhance the temperature sensitivity of PMI. Figure 3 includes a calculation where the temperature sensitivity of phase birefringence is neglected $\left(\Delta \mathrm{n}=2.0 \times 10^{-5}\right.$ was assumed constant for all temperatures). It can be seen that the contribution of both effects, that is dispersion and birefringence is of similar order of magnitude, so neither of them can be neglected. The effective area decreases with the increase of temperature, from $52.2 \mu \mathrm{m}^{2}$ at $20^{\circ} \mathrm{C}$ to $50.3 \mu^{2}$ at $70^{\circ} \mathrm{C}$, however, it has a little impact on the phasematching wavelengths shift. The relative difference of PMI wavelengths taking and neglecting temperature dependence of the effective area is lower than $0.1 \%$.

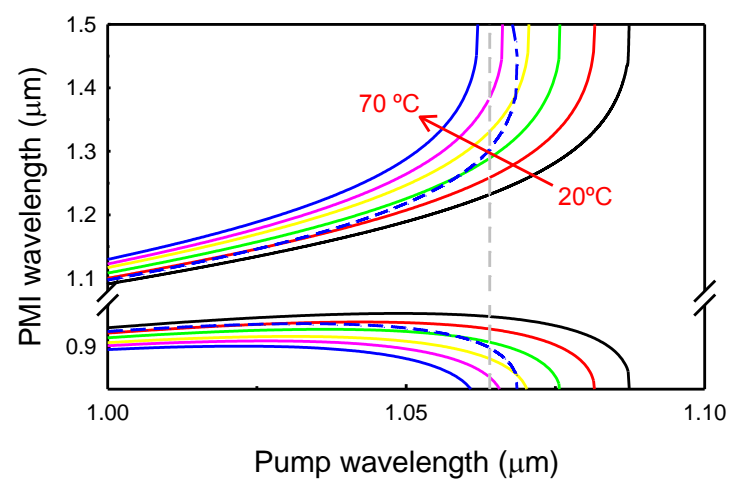

Fig. 3. Phase-matching wavelengths for slow-axis pumping PMI for different temperatures (solid lines). Temperature step between consecutive curves is $10^{\circ} \mathrm{C}$. Phase-matching wavelengths at $70^{\circ} \mathrm{C}$ when the temperature dependence of phase birefringence is neglected (dashed blue line). Vertical grey dashed line indicates the experimental pump wavelength. $\mathrm{P}=1 \mathrm{~kW}$.

The experimental arrangement comprises a passively Qswitched Nd:YAG microchip laser (TEEM Photonics SNP-20F-100) that emits pulses at $1064 \mathrm{~nm}$ of 700 ps duration and few $\mathrm{kW}$ of peak power at a repetition rate of $19.1 \mathrm{kHz}$. The laser emission is linearly polarized with a polarization extinction ratio (PER) of $32 \mathrm{~dB}$. The laser beam was delivered into the fiber under test
(FUT). A half-wave plate (HWP) was used to rotate the polarization plane of the pump beam with respect to the fiber axes.

Fiber sections with length of about $1 \mathrm{~m}$ were infiltrated with ethanol by capillary force and air pressure. Then, they were fusion spliced to a short section of single-mode fiber (injection fiber) and to a multi-mode fiber (collection fiber). To minimize ethanol volatilization during the arc discharge, a section of few $\mathrm{mm}$ long around the fiber cleave was left empty. The temperature of the infiltrated MOFs was varied by immersing them in a temperature controlled thermal bath. The injection fiber was shortened as much as possible to reduce its nonlinear contribution.

Fig. 4 shows an example of the spectrum recorded at room temperature when the input polarization was adjusted to match the slow axis of the fiber. Two strong bands centered at $937 \mathrm{~nm}$ and $1231 \mathrm{~nm}$ generated by PMI are shown. Some other spectral components that correspond to Raman scattering (RS) are also generated. Notice that, in addition to Raman scattered photons generated from the $1064 \mathrm{~nm}$ pump, we can observe Raman bands produced by the PMI bands themselves, which indicates that the intensity of the PMI bands is notable. The peak gain calculated theoretically [7] is $17.3 \mathrm{~m}^{-1}$.

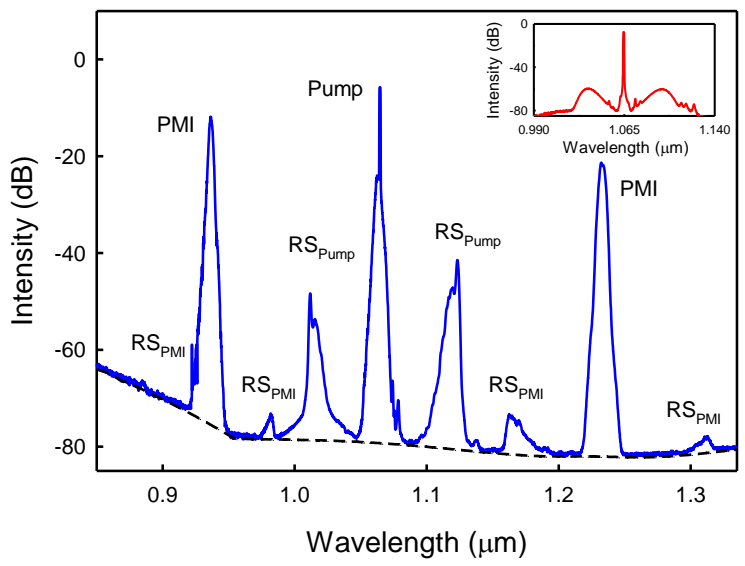

Fig. 4. Spectrum of light at the output of the MOF when the polarization of the $1064 \mathrm{~nm}$ pump was aligned with the MOF slow axis. Fiber length: $1 \mathrm{~m}, \mathrm{P}=4.5 \mathrm{~kW}$. Black dashed line represents the noise level of the optical spectrum analyzer. $\mathrm{RS}_{\text {pump }}$ and $\mathrm{RS}_{\mathrm{PMI}}$ correspond to Raman scattering from the $1064 \mathrm{~nm}$ laser pump and from the PMI bands, respectively.

The polarization of the PMI bands is orthogonal to the polarization of the pump and the main Raman bands. This was checked by using a linear polarizer to analyze the light exiting the MOF. With the orientation of the HWP adjusted to achieve the highest amplitude of the PMI bands, the amplitude of the different spectral components was analyzed as a function of the orientation of the polarizer. When the polarizer was oriented so that the amplitude of the PMI bands was optimized, the amplitude of the rest of spectral components was minimized. From this orientation, rotation of the polarizer produced the reduction of the amplitude of the PMI bands and, simultaneously, the increase of the amplitude of the rest of spectral components. Therefore, the most relevant spectral components, with the exception of the PMI bands can be filtered out by the use of polarizing optics.

The evolution of the spectrum as a function of temperature, between 20 to $65^{\circ} \mathrm{C}$, is shown in Fig. 5 (a). The pump power was 
maintained constant for all temperatures at $5.6 \mathrm{~kW}$ (peak power). Notice that the spectral bands due to Raman scattered photons from the $1064 \mathrm{~nm}$ pump do not change substantially with temperature. As the temperature increases, the anti-Stokes PMI band shifts towards shorter wavelengths, from $937 \mathrm{~nm}$ to $863 \mathrm{~nm}$, and the PMI Stokes band shifts towards longer wavelengths, from $1231 \mathrm{~nm}$ to $1387 \mathrm{~nm}$. Such a tuning range corresponds to a variation of the frequency shift between the pump and a PMI sideband from $1274 \mathrm{~cm}^{-1}$ to $2189 \mathrm{~cm}^{-1}$. The amplitude of the bands tends to decrease for the higher temperatures values. PMI was not observed experimentally for temperatures above $65^{\circ} \mathrm{C}$, as described by the theoretical modelling. Comparison of experimental PMI wavelengths with theoretical calculations is shown in Fig. 5 (b). Good agreement was obtained when the temperature dependence of both birefringence and chromatic dispersion was taken into account. The calculation taking only into account the effect of chromatic dispersion is included in Fig. 5(b). The disagreement with the experimental results is quite large, which highlights the relevance of phase birefringence in this process.

Notice that the PMI wavelengths presented across the paper are obtained from Eq. (1)-(2), thus constant pump power along the fiber is assumed. In some experiments, particularly at high pump powers, the conversion efficiency from the pump to the PMI bands is large and such a condition in not fully accomplished. However, for the fiber of this work the sensitivity of PMI phase-matching wavelengths with pump power is rather small, actually of the same order of the experimental error.
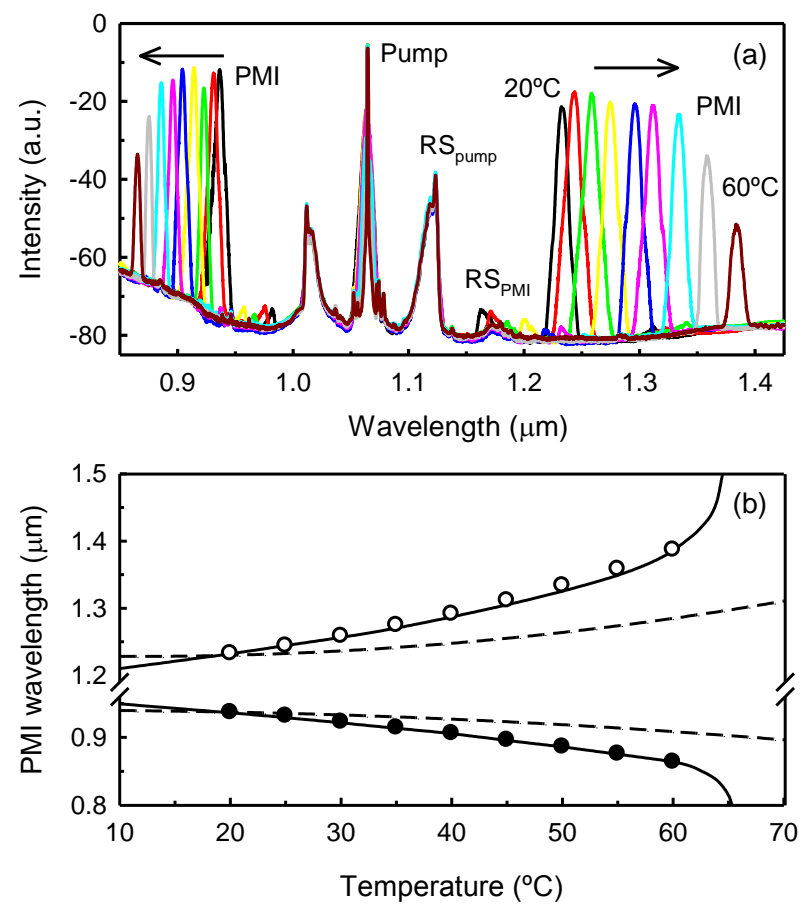

Fig. 5. (a) Light spectrum for different temperatures, from $20^{\circ} \mathrm{C}$ to $65^{\circ} \mathrm{C}$, in steps of $5^{\circ} \mathrm{C}$. Pump peak power: $5.4 \mathrm{~kW}$. (b) PMI wavelengths as a function of temperature. Symbols are experimental data and lines are theoretical calculations. Full calculation (solid line) and calculation in which the temperature dependence of phase birefringence is neglected (dashed line).
In summary, we have demonstrated broadband tuning of widely-spaced PMI spectral bands generated in MOFs filled with ethanol. Owing to the large thermo-optic coefficient of ethanol, moderate changes of temperature produce large changes of ethanol refractive index, which results in changes of fiber chromatic dispersion and birefringence. It is shown that the changes on fiber properties induced by temperature, although slight, can lead to large PMI wavelengths shift provided that the fibers are pumped near the ZDW. In the experimental demonstration, the frequency shift between the pump and PMI sideband ranged from $1274 \mathrm{~cm}^{-1}$ to $2189 \mathrm{~cm}^{-1}$ for a temperature interval from $20^{\circ} \mathrm{C}$ to $60^{\circ} \mathrm{C}$. Good agreement is observed with theoretical calculations of PMI parametric wavelengths obtained from phase and energy conservation.

The results presented in this letter can be of interest for the implementation of dual-wavelength light sources with tunable frequency spacing based on PMI. Several configurations can be foreseen, depending on the polarization requirements of the two spectral lines. As mentioned before, since the polarization of the PMI bands is orthogonal to the polarization of the pump and the main Raman bands, one can clear out the spectrum from most unwanted frequencies by the use of polarizing optics, which can lead to a light source with two equally polarized lines and low background noise compared to light sources based on scalar FWM. On the other hand, a dual-wavelength light source with orthogonally polarized spectral lines can also be implemented utilizing a PMI sideband and the remaining pump.

Funding. This work was supported by the European Union, project IPN-Bio (Ref.: H2020-MSCA-RISE-2019-872049), and by the Generalitat Valenciana of Spain (Ref.: PROMETEO/2019/048).

\section{References}

1. Zhang L., Ultra-Broadly Tunable Light Sources Based on the Nonlinear Effects in Photonic Crystal Fibers. Springer Theses (Springer, Berlin 2015).

2. O. Alibart, J. Fulconis, G. K. L. Wong, S. G. Murdoch, W. J. Wadsworth, and J. G. Rarity, New J. Phys., 8, 67 (2006).

3. T. Gottschall, T. Meyer, M. Baumgartl, C, Jauregui, M, Schmitt, J. Popp, J. Limpert and A. Tünnermann, Laser Photonics Rev. 9, 435-451 (2015).

4. T. Gottschall, T. Meyer, C. Jauregui, M. Schmitt, J. Popp, J. Limpert, A. Tünnermann, Proc. SPIE 9712, 971202 (2016).

5. G. P. Agrawal, Nonlinear Fiber Optics. San Diego, CA, USA: Academic, 2001.

6. S. G. Murdoch, R. Leonhardt, and J. D. Harvey, Opt. Lett., 20, 866-868, (1995).

7. R. J. Kruhlak et al., Opt. Lett., 31, 1379-1381 (2006).

8. A. Loredo-Trejo et al., IEEE Photonics J., 11, 7104208 (2019).

9. M. Baumgartl, M. Chemnitz, C. Jauregui, T. Meyer, B. Dietzek, J. Popp, J. Limpert, and A. Tünnermann, Opt. Express 20, 4484-4493 (2012).

10. L. Velázquez-Ibarra, A. Díez, E. Silvestre, and M. Andrés, Opt. Lett. 41, 2600-2603 (2016).

11. L. Velázquez-Ibarra, A. Díez, E. Silvestre and M. V. Andrés, J. Lightwave Technol., 37, 5722-5726, (2019).

12. Q. Lin and G. P. Agrawal, J. Opt. Soc. Amer. B, vol. 21, pp. 1216-1224, 2004.

13. E. Silvestre, T. Pinheiro-Ortega, P. Andres, J. J. Miret, and A. OrtigosaBlanch, Opt. Lett. 30, 453-455 (2005).

14. S. Kedenburg, M. Vieweg, T. Gissibl, and H. Giessen, Opt. Mater. Express 2, 1588-1611 (2012).

15. Young Ho Kim, Seong Jun Park, Sie-Wook Jeon, Seongmin Ju, Chang-Soo Park, Won-Taek Han, and Byeong Ha Lee, Opt. Express 20, 23744-23754 (2012). 
1. Zhang L., Ultra-Broadly Tunable Light Sources Based on the Nonlinear Effects in Photonic Crystal Fibers. Springer Theses (Springer, Berlin 2015).

2. O. Alibart, J. Fulconis, G. K. L. Wong, S. G. Murdoch, W. J. Wadsworth, and J. G. Rarity, "Photon pair generation using four-wave mixing in a microstructured fibre: Theory versus experiment," New J. Phys., 8, 67 (2006).

3. T. Gottschall, T. Meyer, M. Baumgartl, C, Jauregui, M, Schmitt, J. Popp, J. Limpert and A. Tünnermann, "Fiber-based light sources for biomedical applications of coherent anti-Stokes Raman scattering microscopy," Laser Photonics Rev. 9, 435-451 (2015).

4. T. Gottschall, T. Meyer, C. Jauregui, M. Schmitt, J. Popp, J. Limpert, A. Tünnermann, "Four-wave mixing based light sources for real-world biomedical applications of coherent Raman microscopy," Proc. SPIE 9712, 971202 (2016)

5. G. P. Agrawal, Nonlinear Fiber Optics. San Diego, CA, USA: Academic, 2001.

6. S. G. Murdoch, R. Leonhardt, and J. D. Harvey, "Polarization modulation instability in weakly birefringent fibers," Opt. Lett., 20, 866-868, (1995).

7. R. J. Kruhlak et al., "Polarization modulation instability in photonic crystal fibers," Opt. Lett., 31, 1379-1381 (2006).

8. A. Loredo-Trejo et al., "Polarization modulation instability in all-normal dispersion microstructured optical fibers with quasi-continuous pump," IEEE Photonics J., 11, 7104208 (2019).

9. M. Baumgartl, M. Chemnitz, C. Jauregui, T. Meyer, B. Dietzek, J. Popp, J. Limpert, and A. Tünnermann, "All-fiber laser source for CARS microscopy based on fiber optical parametric frequency conversion," Opt. Express 20, 4484-4493 (2012).

10. L. Velázquez-Ibarra, A. Díez, E. Silvestre, and M. Andrés, "Wideband tuning of four-wave mixing in solid-core liquid-filled photonic crystal fibers," Opt. Lett. 41, 2600-2603 (2016).

11. L. Velázquez-Ibarra, A. Díez, E. Silvestre and M. V. Andrés, "Tunable FourWave Mixing Light Source Based on Photonic Crystal Fibers With Variable Chromatic Dispersion," J. Lightwave Technol., 37, 5722-5726, (2019).

12. Q. Lin and G. P. Agrawal, "Vector theory of four-wave mixing: polarization effects in fiber-optic parametric amplifiers," J. Opt. Soc. Amer. B, vol. 21, pp. 1216-1224, 2004.

13. E. Silvestre, T. Pinheiro-Ortega, P. Andres, J. J. Miret, and A. OrtigosaBlanch, "Analytical evaluation of chromatic dispersion in photonic crystal fibers," Opt. Lett. 30, 453-455 (2005).

14. S. Kedenburg, M. Vieweg, T. Gissibl, and H. Giessen, "Linear refractive index and absorption measurements of nonlinear optical liquids in the visible and near-infrared spectral region," Opt. Mater. Express 2, 15881611 (2012)

15. Young Ho Kim, Seong Jun Park, Sie-Wook Jeon, Seongmin Ju, Chang-Soo Park, Won-Taek Han, and Byeong Ha Lee, "Thermo-optic coefficient measurement of liquids based on simultaneous temperature and refractive index sensing capability of a two-mode fiber interferometric probe," Opt. Express 20, 23744-23754 (2012). 